Fixed Point Theory, 21(2020), No. 2, 707-714

DOI: $10.24193 /$ fpt-ro.2020.2.50

http://www.math.ubbcluj.ro/ nodeacj/sfptcj.html

\title{
A GENERALIZATION OF AMINI-HARANDI'S FIXED POINT THEOREM WITH AN APPLICATION TO NONLINEAR MAPPING THEORY
}

\author{
KÜBRA ÖZKAN*, UTKU GÜRDAL** AND ALI MUTLU*** \\ * Manisa Celal Bayar University, Department of Mathematics, 45140 Manisa Turkey \\ E-mail: kubra.ozkan@hotmail.com, kubra.ozkan@cbu.edu.tr (Corresponding author) \\ ** Mehmet Akif Ersoy University, Department of Mathematics, 15030, Burdur Turkey \\ E-mail: utkugurdal@gmail.com \\ *** Manisa Celal Bayar University, Department of Mathematics, 45140 Manisa Turkey \\ E-mail: abgamutlu@gmail.com
}

\begin{abstract}
We introduce a new fixed point theorem on complete metric spaces, which generalizes some former results, and we apply this to obtain a surjectivity theorem for Gâteaux differentiable mappings between Banach spaces.
\end{abstract}

Key Words and Phrases: Complete metric space, fixed point, Gâteaux derivative.

2010 Mathematics Subject Classification: 54E50, 54H25, 47H10.

\section{REFERENCES}

[1] Ö. Acar, İ. Altun, Some generalizations of Caristi type fixed point theorem on partial metric spaces, Filomat, 26(2012), no. 4, 833-837.

[2] R.P. Agarwal, M.A. Khamsi, Extension of Caristi's fixed point theorem to vector valued metric space, Nonlinear Anal., 74(2011) 141-145.

[3] C. Alegre, J. Marin, A Caristi fixed point theorem for complete quasi-metric spaces by using mw-distances, Fixed Point Theory, 19(2018), no. 1, 25-32.

[4] S. Al-Homidan, Q.H. Ansari, J.C. Yao, Some generalizations of Ekeland-type variational principle with applications to equilibrium problems and fixed point theory, Nonlinear Anal., 69(2008), no. 1, 126-139.

[5] A. Amini-Harandi, Some generalizations of Caristi's fixed point theorem with applications to the fixed point theory of weakly contractive set-valued maps and the minimization problem, Nonlinear Anal., 72(2010), no. 12, 4661-4665.

[6] Q.H. Ansari, Vectorial form of Ekeland-type variational principle with applications to vector equilibrium problems and fixed point theory, J. Math. Anal. Appl., 334(2007), no. 1, 561-575.

[7] J.S. Bae, Fixed point theorems for weakly contractive multivalued maps, J. Math. Anal. Appl., 284(2003), no. 2, 690-697.

[8] S. Banach, Sur les opérations dans les ensembles abstraits et leur application aux équations intégrales, Fund. Math., 3(1922) 133-181.

[9] J. Caristi, Fixed point theorems for mappings satisfying inwardness conditions, Trans. Amer. Math. Soc., 215(1976), 241-251. 
[10] S.H. Cho, Some generalizations of Caristi's fixed point theorem with applications, Int. J. Math. Analysis, 7(2013), no. 12, 557-564.

[11] L. Ćirić, A generalization of Caristi's fixed point theorem, Math. Pannonica, 3(1992), no. 2, 51-57.

[12] D. Downing, W.A. Kirk, A generalization of Caristi's theorem with applications to nonlinear mapping theory, Pacific J. Math., 69(1977), 339-346.

[13] I. Ekeland, Remarques sur les problémes variationnels I, C.R. Acad. Sci. Paris, 276(1972), 1057-1059.

[14] M.R. Gâteaux, Sur les fonctionnelles continues et les fonctionnelles analytiques, C.R. Acad. Sci. Paris, 157(1913), no. 65, 325-327.

[15] E. Karapinar, Generalizations of Caristi Kirk's theorem on partial metric spaces, Fixed Point Theory Appl., 2011(2011), no. 1, 4.

[16] M.A. Khamsi, Remarks on Caristi's fixed point theorem, Nonlinear Anal., 71(2009), no. 1-2, 227-231.

[17] A. Latif, Generalized Caristi's fixed point theorems, Fixed Point Theory Appl., 2009(2009), no. 1, ID 170140 (7 pages).

[18] S. Park, On generalizations of the Ekeland-type variational principles, Nonlinear Anal., 39(2000), no. 7, 881-889.

[19] A. Petruşel, Caristi type operators and applications, Bul. Ştiinţ. Univ. Politeh. Timiş. Ser. Mat. Fiz., XLVIII(2002), no. 3, 297-302.

[20] I.A. Rus, A. Petruşel, G. Petruşel, Fixed Point Theory, Cluj University Press, 2008.

[21] T. Suzuki, Generalized Caristi's fixed point theorems by Bae and others, J. Math. Anal. Appl., 302(2004), no. 2, 502-508.

[22] W.C. Weston, A characterization of metric completeness, Proc. Amer. Math. Soc., 64(1977), no. $1,186-188$.

Received: June 18, 2018; Accepted: January 10, 2019. 